\title{
Markers of the pre-metastatic niche "knock on the door" of metastasis-free cervical lymph nodes in patients with oral cancer
}

\section{Vered, Marilena}

2019-11

Vered, M , Shnaiderman-Shapiro , A , Schiby , G, Zlotogorski-Hurvitz , A, Salo , T \& Yahalom , R 2019 , ' Markers of the pre-metastatic niche "knock on the door" of metastasis-free cervical lymph nodes in patients with oral cancer ', Acta histochemica : a journal of structural biochemistry, cell and tissue imaging , vol. 121 , no. 8,151447 . https://doi.org/10.1016/j.acthis.

http://hdl.handle.net/10138/320981

https://doi.org/10.1016/j.acthis.2019.151447

cc_by_nc_nd

acceptedVersion

Downloaded from Helda, University of Helsinki institutional repository.

This is an electronic reprint of the original article.

This reprint may differ from the original in pagination and typographic detail.

Please cite the original version. 


\section{Markers of the pre-metastatic niche "knock on the door" of metastasis-free cervical lymph nodes in patients with oral cancer}

Marilena Vered ${ }^{\mathrm{a}, \mathrm{b},{ }^{*}}$, Anna Shnaiderman-Shapiro ${ }^{\mathrm{a}}$, Ginette Schiby ${ }^{\mathrm{b}}$, Ayelet ZlotogorskiHurvitz $^{\mathrm{a}, \mathrm{c}}$, Tuula Salo ${ }^{\mathrm{d}, \mathrm{e}}$, Ran Yahalom ${ }^{\mathrm{f}}$

${ }^{a}$ Dept. Oral Pathology, Oral Medicine and Oral Radiology, School of Dental Medicine, Tel Aviv University, Tel Aviv, Israel

b Institute of Pathology, The Chaim Sheba Medical Center, Tel Hashomer, Ramat Gan, Israel 'Dept. Oral and Maxillofacial Surgery, The Rabin Medical Center, Beilinson Campus, Petah Tikva, Israel

${ }^{d}$ Cancer and Translational Research Unit, University of Oulu and MRC, Oulu University Hospital, Oulu, Finland

'Oral and Maxillofacial Diseases, Clinicum, University of Helsinki, Helsinki, and HUSLAB, Helsinki University Hospital, Helsinki, Finland

fDept. Oral and Maxillofacial Surgery, The Chaim Sheba Medical Center, Tel Hashomer, Ramat Gan, Israel

${ }^{*}$ Corresponding author:

Email:mvered@post.tau.ac.il

Tel: +972-3-6409305

Fax: +972-3-6409250

Keywords: oral cancer, cervical lymph nodes, pre-metastatic niche

\section{Abbreviations:}

CAP - capsule; ECM extracellular matrix; FN fibronectin; LN - lymph node; LOX-lysyl oxidase; M M P-9 - matrix metalloproteinase 9; MS - medullary sinus; PM N - pre-metastatic niche; SCS - sub-capsular sinus; TME tumor microenvironment; VEGFR- 1 - vascularendothelial growth factor receptor 1 ; 


\section{Abstract}

Aim: To assess expression of some markers of the pre-metastatic niche (PMN) in lymph nodes (LNs) of oral cancer patients.

Materials: LNs from metastatic-free neck dissections ( $\mathrm{LNO} / \mathrm{NO}, \mathrm{N}=43$ ) and metastatic-free LNs in the vicinity of metastasis-containing $L N s \quad(L N O / N+, N=30)$ were immunehistochemically stained for lysyl oxidase (LOX), fibronectin (FN), vascular-endothelial growth factor receptor (VEGFR)-1 and matrix metalloproteinase (MMP)-9. Staining was assessed as 0 (no or weak staining), 1 (strong stain in $25 \%$ cells or extracellular area), 2 (same as 1 but in up to $50 \%$ ) and 3 (same as 1 but in > than $50 \%$ of cells/area). Assessment was performed in the lymph node capsule (CAP), sub-capsular sinus (SCS) and medullary sinus (MS). In addition, sections were stained with picrosirius red and examined with polarized microscopy for assessing the distribution of polarization colors of the collagen fibers in the LN capsular area.

Results: All examined LNs were positive for markers of the PMN. In general, the distribution and intensity of the immunoreactivity was similar between the LNO/NO and LNO/N+, with only a few differences regarding expression of LOX in the capsule $(p=0.002)$ and VEGFR1 and MMP9 in the SCS ( $p=0.023$ and $p<0.001$, respectively). Picrosirius red stain and polarized microscopy revealed a disrupted arrangement and distribution of the collagen fibers in both LNO/NO and LNO/N+.

Conclusion: Markers for PMN were shown for the first time to be expressed in cervical LNO/NO from patients with oral cancer, suggesting the increased permissive pathway remotely paved by the primary oral tumor for the incoming metastatic cells. 


\section{Introduction}

The majority of cancer-related deaths are caused by the metastatic disease rather than by the primary tumor, and oral cancer (i.e., oral squamous cell carcinoma, OSCC) is no exception. The 5-year survival of oral cancer patients is $~ 50 \%$ (Ferlay et al., 2015) and presence of regional metastatic disease further decreases it by half for each stage of the disease (Patel et al, 2011).

While cancer research has traditionally focused on the malignant cells, more recent studies have provided evidence on the dependency of the primary tumors on their stroma (i.e., tumor microenvironment, TME) and the critical role that the stroma has on the tumor development, progression and metastatic spread (Sleeman, 2012; Spill et al. 2016). This tumor-TME co-evolution seems to be valid also for the secondary, metastatic sites. Survival and proliferation of tumor cells as successful metastatic outgrowths require a receptive TME at the destination site. The term "pre-metastatic niche"(PMN) has been coined to describe the process of creating a TME that is conducive for tumor cell adhesion and invasion at the putative metastatic organ/site (Psaila and Lyden, 2009). There seems to be sites that endogenously provide all or most components of the PMN, while others need substantial remodeling in order to become fully competent for the arrival of the disseminated tumor cells (Sleeman 2012). The remodeling is assumed to be remotely induced, at least in part, by growth factors secreted from the primary tumor that exert their impact on the PMN as well as on the bone marrow, from which specific progenitor cells are mobilized and recruited to the PMN (Peinado et al. 2011; Psaila and Lyden, 2009; Sleeman, 2012). Key factors that participate in the initiation of the PMN include fibronectin (FN), lysyl oxidase (LOX), bone marrow-derived cells with a phenotype positive for vascular endothelial growth factor receptor (VEGFR)-1 and matrix metalloproteinase (MMP)-9. As a consequence of the action of LOX and MMP9 extensive remodeling of the extracellular matrix (ECM) occurs.

The concept of PMN has been studied primarily on breast cancer and melanoma as primary tumors, and the lungs as the destination organ for metastasis (Peinado et al. 2011; Psaila and Lyden, 2009; Sleeman, 2012). The PMN in oral cancer has been barely investigated, so far. Only a few studies have referred to lymphangiogenesis within the cervical LNs as an indicator of PMN (Mayorca-Guiliani et al. 2012; Wakisaka et al. 2015).

We recently conducted an extensive histomorphologic study on the architectural features of cervical lymph nodes (LNs) from patients with OSCC, including features such as the capsular thickness, degree of dilatation of the sub-capsular and medullary sinuses, the lobular structure and the reactivity status of the lymphoid follicles (Vered et al. 2014). In that study we compared metastatic-free LNs (LNO) from neck dissections in which all LNs were metastatic-free (LNO/NO) to LNO selected from neck dissections that contained a number of metastatic-loaded $\mathrm{LNs}(\mathrm{LNO} / \mathrm{N}+)$ and found that both types of $\mathrm{LNs}$ showed similar architectural changes. Therefore, we assumed that the presence of metastatic disease is not necessary to induce changes in the adjacent LNs and that those changes found in LNO/NO could reflect the remote impact of the primary oral tumor on the putative metastatic site, conforming to the concept of PMN. It was the aim of the present study to examine whether recognized molecular markers of PMN initiation are present in LNO from OSCC patients.

\section{Material and Methods}

$\mathrm{LNO} / \mathrm{NO}(\mathrm{N}=43)$ and $\mathrm{LNO} / \mathrm{N}+(\mathrm{N}=30)$ were selected from the $\mathrm{LN}$ series described in our previous study (Vered et al. 2014). Since most of the metastatic LNs were found at cervical dissection levels II and III, all LNs in this study were selected from these levels. 
$\mathrm{LNO} / \mathrm{N}+$ were always located in the vicinity of metastasis-loaded LNs. The study was conducted with the approval of the IRB, The Chaim Sheba Medical Center.

Immunohistochemical stains were prepared on $3 \mu \mathrm{m}$-thick sections using antibodies against LOX (1:100, Proteintech, Manchester, UK; positive control - placenta), FN (1:250, Abcam, Cambridge, UK; control tissue - kidney), VEGFR-1 (1:100, Acris, Herford, Germany; control tissue breast carcinoma) and MMP-9 (1:100, Acris, Herford, Germany; control tissue ovarian carcinoma). Negative controls were obtained by omitting the primary antibody.

Staining was assessed as 0 (no or weak staining), 1 (strong stain in $25 \%$ cells or extracellular area), 2 (same as 1 but in up to 50\%) and 3 (same as 2 but in > than $50 \%$ of cells/area). Assessment was performed in the lymph node capsule (CAP), sub-capsular sinus (SCS) and medullary sinus (MS). In regard to LOX, we have considered the SCS together with the immediately located cortex as staining seemed to generate a continuum in these two adjacent compartments. Results were presented as the frequency of immunoreactivity of each marker for each score of the LNO/NO and LNO/N+.

Collagen molecules are rich in basic amino acids, which strongly react with acidic dyes, like sirius red. Binding of this dye promotes an enhancement of the natural collagen bundles birefringence under polarized light (Junqueira et al. 1979). Histochemical staining process was done with picrosirius red (PSR, $0.1 \%$ sirius red in saturated aqueous picric acid). Eight micron-thick sections were incubated for $30 \mathrm{~min}$ with PSR. Sections were studied by a light microscope under polarized light (Olympus BX-50, Tokyo, Japan). Original photomicrographs of 3 representative capsular areas of each $L N$ were taken using a camera mounted on the microscope (Olympus DP70, Tokyo, Japan). Photomicrographs of the same area were taken under identical conditions (exposure time, plane of microscope stage, position of polarizer) after $90^{\circ}$ stage rotation. Photomicrographs were saved as jpeg files that were subsequently copied onto power-point (PPT) slides, each slide showing side-byside the original and the $90^{\circ}$-rotated counterpart picture. A grid composed of 10 doted parallel lines, with an equal distance between any 2 following lines, was superimposed on each picture. Those collagen bundles that overlapped the grid lines on the original picture were identified and their color was recorded and then compared to the counterpart, $90^{\circ}$ rotated picture. Changes in polarization colors of the collagen bundles between the original to the rotated picture were recorded: green-to-yellow, green-to-orange/red, yellow-toorange/red and vice versa. In addition, events of disappearance or appearance of polarization colors that were generated by the $90^{\circ}$ rotation were also recorded. The percent of those collagen bundles that changed colors from the total number of bundles that was recorded for each case was calculated and results were presented as the mean percent number of collagen fibers with change of color for the LNO/NO and LNO/N+.

Statistical analysis was performed by crosstabs (immunohistochemical stains) and Mann-Whitney test (PSR stain) using the SPSS, version 21 (Chicago IL, USA). Statistical significance was set at $p<0.05$.

\section{Results}

LOX1

Staining was mainly intracellular and this included spindle cells within the capsule, epithelioid cells within the sub-capsular sinuses and lymphocytes within the adjacent cortex. 
LNO/NO: the most common score was 1 for the CAP and MS and score 2 for the SCS. When grouped together, scores 1 and 2 were most frequent for the CAP, SCS and MS (63\%, 77\% and $65 \%$, respectively) (Fig. $1 \mathrm{~A}, \mathrm{~B}$ ). Score 3 was uncommon in all the examined LN compartments.

$\mathrm{LNO} / \mathrm{N}+$ : although the same pattern of score distribution was found as in LNO/NO, there was a shift toward scores 2 and 3 , in particular in regard to CAP ( $p=0.002)$ (Fig. 2A).

\section{FN}

LNO/NO: the CAP was the main LN compartment to demonstrate strong and diffuse staining of score 3. SCS and MS were positively stained usually in the range of scores 1 and 2 (Fig. $1 D, E)$.

LNO/N+: staining in the CAP was more evenly distributed between scores 1 and 3 in comparison to LNO/NO. The pattern of staining distribution in the SCS and MS was similar to that seen in LNO/NO. Altogether, no significant differences were found between $\mathrm{LNO} / \mathrm{N}+$ and LNO/NO ( $p>0.05$ ) (Fig. 2B).

\section{VEGFR1}

LNO/NO: positively stained cells concentrated in the area of SCS and especially that of the MS (Fig. 1G,H).

LNO/N+: the most remarkable change compared to LNO/NO was seen in regard to the SCS, with a 2.5-fold increase in the frequency of positively stained cells of score 1 ( $p=0.023$ ) (Fig. 2C).

MMP9

LNO/NO: most positively-stained cells in SCS and MS were in the range of scores 1 and 2 (Fig. $1 \mathrm{~J}, \mathrm{~K}$ and Fig. 2D). Although the CAP area showed a similar trend, score 2 was considerably less frequent than in SCS and MS.

LNO/N+: the main difference from LNO/NO was the lower frequency of stained cells in the SCS $(p<0.001)$ (Fig. 2D).

PSR

Under polarized light, the capsule of both LNO/NO and LNO/LN+ presented a similar pattern that was composed of an admixture of thick orderly arranged collagen bundles and fibers together with haphazardly dispersed, remarkably thin, irregular and fragmented fibrils (Fig. 3). In term of the intensity, there were foci of highly birefringent collagen fibers alongside those showing weak birefringence. The birefringence spectrum ranged between greenyellow-orange and red colors. The percent of change in polarization colors between the perpendicular positioned photomicrographs was $35.8 \pm 16.2 \%$ for the LNO/NO and $37.9 \pm 15.2 \%$ for the $\mathrm{LNO} / \mathrm{N}+(\mathrm{p}>0.05)$.

\section{Discussion}

Our study is the first to investigate the expression of markers for the pre-metastatic niche in regional LNs in oral cancer. We found positive expression of the selected markers in all examined LNO. These results suggest that the concept that OSCC patients who are free of regional (cervical) metastases have a tumor constrained to the oral cavity does not hold true any longer. LNO/NO are primed before the arrival of the metastatic cells, most probably as a result of the systemic effects of different factors secreted by the primary oral tumor. Although several differences were found, the degree of priming of the LNO/NO was, in general, similar to that of $\mathrm{LNO} / \mathrm{N}+$, pointing to the fact that adapting the soil of the LNs does 
not require spatial closeness to the tumor but can be achieved in a remote manner. In line with this, it has been claimed that factors from the primary tumor present in the circulation are responsible for generation of promoting conditions at potential target PMN sites (Sleeman, 2012). This has gained support by our identification of VEGFR1+ in cervical LNs of patients who were diagnosed only with an oral cancer and free of cervical metastatic disease (i.e., LNO/NO). On the other hand, the presence of some PMN markers may indicate the endogenous expression of those markers in pre-existing 'inducible niches' of LNs which attracts the circulating cancer cells (Psaila et al. 2009). Fig. 4 is a schematic illustration of the formation of PMNs in cervical lymph nodes of oral cancer patients, based on our findings and those presented in previous studies (Psaila and Lyden, 2009; Psaila et al. 2009).

LOX and clusters of bone marrow-derived hematopoietic progenitor cells (i.e., VEGFR1+) are crucial in initiating the assembly of other components of the PMN (Psaila and Lyden, 2009). Directing cell migration and metastasis depends on the physical features of the ECM which should provide mechanical stiffness, fiber alignment and crosslinking. Secreted LOX is in charge for collagen cross-linking and the interaction with FN enhances its catalytic activity, leading to increased stiffness of the ECM (Barker et al. 2011). In the examined LNs, expression of LOX and FN was found to coincide in all compartments. LOX (as well as FN) most likely originates from LN resident activated fibroblasts, other local stromal cells, or even may be carried from the primary oral tumor and laid by metastatic cells that transiently pass through the LNs (Psaila and Lyden, 2009).

In the present series of LNs we could not identify secreted LOX, its expression being intracellular, mostly within spindle-shaped cells in the LN fibrous capsule as well as in lymphocytes in the adjacent cortical follicles. It has been suggested that intracellular LOX may catalyze globular proteins such as histones with a result of preserving chromosomal stability, an effect that may be associated with the anti-tumoral activity of LOX (Barker et al. 2011). However, it seems that the pro-tumoral impact of LOX prevails even when it is expressed intracellularly. In a study on mammary glands, it has been shown that when these glands were conditioned with LOX-expressing fibroblasts they became harder in consistency compared to glands containing LOX-negative fibroblasts, assumedly because LOX-positive fibroblasts enhanced the deposition of fibrillar and linearized (cross-linked) collagen (Levental et al. 2009). In addition, it has been demonstrated that T-lymphocytes also express LOX and that they play a key role in the modulation of LOX-dependent collagen maturation (Zibadi et al. 2010; Yu et al. 2010).

Continuous tissue remodeling makes the relationship between the production of collagen and its degradation critical to the maintenance of tensile strength (Borges et al 2007). Our observation regarding the absence of secreted LOX could also be related to the fact that the ECM within the LNs has already reached peak stiffness and it has shifted into a protease-dependent remodeling stage (Spill et al 2016). It appears that LOX-mediated remodeling of the ECM acts in a synergistic manner with MMPs (Barker et al 2011). MMP9, a an important factor in ECM remodeling, is assumed to originate from VEGFR1+-bone marrow-derived cells, from activated local fibroblasts, endothelial cells as well as macrophages (Peinado et al. 2011; Psaila and Lyden, 2009). The end-point of the enzymatic remodeling of ECM, including the gelatinolytic activity of MMP9, would be the formation of a fragmented collagen meshwork, the effect of which would be an increased potential for metastatic engraftment. Using picro-sirius red and polarized microscopy we were able to demonstrate the outcome of enzymatic activity on the collagen fibers in the LN capsule. 
Collagen molecules are rich in basic amino acids and as such they react strongly and specifically with anionic dyes, like sirius red. Many dye molecules align in parallel to the long axis of each collagen molecule. When collagen molecules are arranged in orderly oriented aggregates, the sirius red dye promotes enhancement of its normal birefringence (Borges et al. 2007). The degree of polymerization of the collagen molecules and the three-dimensional organization of the fibers can affect the amount of dye uptake. When sirius red-stained sections are analyzed under polarization light, the collagen fibers normally appear regularly arranged, forming thick bundles of continuous and densely packed fibers with a yellow and/or red bright birefringent while the thin fibers display a greenish color and weak birefringence. Under conditions that impair the physical aggregation of the collagen molecules, the collagen appears as fragmented and irregularly separated small structures with weak birefringent. This corresponds to the overall appearance of the capsular collagen fibers revealed by sirius red stain and polarized light in both LNO/NO and LNO/N+. Furthermore, when the collagen birefringence was examined after $90^{\circ}$ rotation of the microscope stage the amount of change in polarization colors was similar in LNO/NO and $\mathrm{LNO} / \mathrm{N}+$. This finding may imply that the collagen fibers within the capsule of both types of LNs reflect the same pattern of molecular order, organization and heterogeneity of orientation (Lattouf et al. 2014).

In conclusion, this is the first study that investigated the molecular properties of cervical lymph nodes in patients with oral cancer in terms of the expression of markers related to the formation of the PMN. Limitations presented by this kind of study lie in the lack of a "real" control group of "healthy" LNs. On the other hand, including metastatic LNs would already show established changes induced by the tumor. Therefore, selecting nonmetastatic LNs in the vicinity of metastatic LNs seemed to be the most suitable available control. According to the present results, we suggest that the tumor within the oral cavity has the ability to induce molecular changes in metastatic-free cervical lymph nodes (i.e., LNO/NO), similar to what has been previously reported for other types of cancers, such as breast and melanoma, and their corresponding putative sites of pre-metastatic niche formation, especially the lungs. In general, the changes that were found in LNO/NO were comparable to those found in lymph nodes that were located in the vicinity of metastatic lymph nodes (i.e., LNO/N+). These findings can indicate that it was the primary tumor from the oral cavity that dictated the molecular changes toward a pre-metastatic niche in the "remote" cervical lymph nodes and that the contribution of the metastatic tumor on the "close" lymph nodes was usually only of a less extent.

\section{Acknowledges}

The study was supported by the Ernest and Tova Turnheim Clinical Research Fund in Dentistry, Tel Aviv University. The funding source was not scientifically involved in the study.

The authors make a special tribute to late Prof. Dan Dayan, for his contribution to the initial design of the study.

The authors would like to thank Hana Vered (Israel) and Tanja Kuusisto (Finland) for technical assistance. 


\section{References}

Barker, H.E., Cox, T.R., Erler, J.T., 2012. The rationale for targeting the LOX family in cancer. Nat. Rev. Cancer. 12, 540-552.

Borges, L.F., Gutierrez, P.S., Marana, H.R., Taboga, S.R., $2007 . \quad$ Picrosiriuspolarization staining method as an efficient histopathological tool for collagenolysis detection in vesical prolapse lesions. Micron. 38, 580583.

Ferlay, J., Soerjomataram, I.I., Dikshit, R., Eser, S., Mathers, C., Rebelo, M., Parkin, D.M., Forman, D., Bray, F., 2015. Cancer incidence and mortality worldwide: sources, methods and major patterns in GLOBOCAN 2012. Int. J. Cancer. 136, E359-386.

Junqueira, L.C., Bignolas, G., Brentani, R.R., 1979. Picrosirius staining plus polarization microscopy, a specific method for collagen detection in tissue sections. Histochem. J. 11, 447-455.

Lattouf, R., Younes, R., Lutomski, D., Naaman, N., Godeau, G., Senni, K., Changotade, S., 2014. Picrosirius red staining: a useful tool to appraise collagen networks in normal and pathological tissues . J. Histochem. Cytochem. 62, 751758.

Levental, K.R., Yu. H., Kass, L., Lakins, J.N., Egeblad, M., Erler, J.T., Fong, S.F., Csiszar, K., Giaccia, A., Weninger, W., Yamauchi, M., Gasser, D.L., Weaver, V.M. 2009. Matrix crosslinking forces tumor progression by enhancing integrin signaling. Cell. 139, 891906.

Mayorca-Guiliani, A.E., Yano, H., Nakashiro, K., Hamakawa, H., Tanaka, J. 2012. Premetastatic vasculogenesis in oral squamous cell carcinoma xenograft-draining lymph nodes. Oral. Oncol. 48, 663670.

Patel, V., Marsh, C.A., Dorsam, R.T., Mikelis, C.M., Masedunskas, A., Amornphimoltham, P., Nathan, C.A., Singh, B., Weigert, R., Molinolo, A.A., Gutkind, J.S. 2011. Decreased lymphangiogenesis and lymph node metastasis by mTOR inhibition in head and neck cancer. Cancer. Res. 71, 71037114.

Peinado, H., Lavotshkina, S., Lyden, D. 2011. The secreted factors responsible for pre-metastatic niche formation: Old sayings and new thoughts. Semin. Cancer. Biol. 21, 139 146.

Psaila, B., Lyden, D. 2009. The metastatic niche: adapting the foreign soil. Nat. Rev. Cancer. 9, 285-293.

Sleeman, J. 2012. The metastatic niche and stromal progression. Cancer. Metastasis. Rev. 31, 429440.

Spill, F., Reynolds, D.S., Kamm, R.D., Zaman, M.H. 2016. Impact of the physical microenvironment on tumor progression and metastasis. Curr. Opinion. Biotech. 40, 4148.

Vered, M., Schiby, G., Schnaiderman-Shapiro, A., Novikov, I., Bello, I.O., Salo, T., Rytkonen, A., Kauppila, J.H., Dobriyan, A., Yahalom, R., Taicher, S., Dayan, D. 2014. Key 
architectural changes in tumor-negative lymph nodes from metastatic-free oral cancer patients are valuable prognostic factors. Clin. Exp. Metastasis 31, 327338.

Wakisaka, N., Hasegawa, Y., Yoshimoto, S., Miura, K., Shiotani, A., Yokoyama, J., Sugasawa, M., Moriyama-Kita, M., Endo, K., Yoshizaki, T. 2015. Primary tumor-secreted lymphangiogenic factors induce pre-metastatic lymphovascular niche formation at sentinel lymph nodes in oral squamous cell carcinoma. PLOS ONE 10, e0144056.

Yu, Q., Vazquez, R., Zabadi, S., Watson, R.R., Larson, D.F. 2010. T-lymphocytes mediate left ventricular fibrillar collagen crosslinking and diastolic dysfunction in mice. Matrix. Biol. 29, 511518.

Zibadi, S., Vazquez, R., Larson, D.F., Watson, R.R. 2010. T lymphocyte regulation of lysyl oxidase in diet-induced cardiac fibrosis. Cardiovasc. Toxicol. 10, 190-198. 


\section{Legend to figures}

Fig. 1 Representative sections of LNO/NO and LNO/N+ immunostained for LOX (A-C), fibronectin (D-F), VEGFR-1 (G-I) and MMP-9 (J-L). Scale bar $=200 \mu$

Fig. 2 Graphical presentation of the frequency (\%) and intensity (score) of the expression of LOX (A), fibronectin (B), (VEGFR)-1 (C) and MMP-9 (D) in LNO/NO and LNO/N+ as a factor of lymph node compartments: capsule (Cap), sub-capsular sinus (SCS) and medullary sinus (MSC).

Fig. 3

Samples of lymph node capsule stained with picrosirius red and observed under polarized light. The section background appears black and the collagen fibers are red, yellow and green. A represents a metastatic-free lymph node from a metastatic-free neck dissection (LNO/NO) and B represents the same section observed after microscope stage rotation of $90^{\circ}$. Arrows point to collagen bundles that changed polarization colors after the rotation. The overall pattern of the collagen fibers is that of a disrupted arrangement and distribution. $\mathrm{C}$ represents a metastatic-free lymph node from a metastatic-positive neck dissection $(\mathrm{LNO} / \mathrm{N}+)$ and $\mathrm{D}$ represents the same section observed after microscope stage rotation of $90^{\circ}$. Arrows point to collagen bundles that changed polarization colors after the rotation. $\mathrm{E}$ and $\mathrm{F}$ show conspicuous decrease in birefringence intensity ( $\mathrm{F}$ after $90^{\circ}$ rotation) indicating collagenolysis. A, B, C and D scale bar $=50 \mu ; E$ and F scale bar $=100 \mu$

Fig. 4

A. Schematic illustration of the molecular cross-talk pathways among the primary tumor, the bone marrow and the site of the pre-metastatic niche. B. The priming of the pre-metastatic site enables engulfment of the metastatic cells and their conversion into a micro-metastasis. CXCR4 CX-chemokine receptor 4; FN- fibronectin, LOX-1 lysyl oxidase-1; MMP-9 matrix metallo-proteinase-9; PIGF placental growth factor; SDF1 - stromal-derived growth factor$1 ;$ SAA3 - $\quad$ - transforming growth factor- - tumor necrosis factor alpha; VEGF-A - vascular endothelial growth factor $A$

- Collagen fibers 


\section{Highlights:}

- Markers of pre-metastatic niche are present in metastasis-free cervical lymph nodes

- Cervical lymph nodes are primed before the arrival of the metastatic cells

- Priming of lymph nodes involves extensive remodeling of extracellular matrix 